\title{
Puberdade em touros Nelore criados em pasto no Brasil: características corporais, testiculares e seminais e de índice de capacidade andrológica por pontos
}

\author{
[Puberty in Nellore bulls raised at pasture in Brazil: body, testicular and seminal \\ characteristics and breeding soundness evaluation] \\ G.E. Freneau ${ }^{1}$, V.R. Vale Filho ${ }^{2}$, A.P. Marques Jr. ${ }^{2}$, W.S. Maria ${ }^{3}$ \\ ${ }^{1}$ Universidade Federal de Goias \\ Caixa Postal 131 \\ Goiânia, GO \\ ${ }^{2}$ Escola de Veterinária - UFMG - Belo Horizonte, MG \\ ${ }^{3}$ Fundação Ezequiel Dias - Belo Horizonte, MG
}

\begin{abstract}
RESUMO
Foram utilizados 23 touros da raça Nelore entre os 10 e 20 meses de idade, para caracterizar o desenvolvimento puberal. Os animais foram criados em condições extensivas com alimentação em pasto e suplementação mineral. A cada duas ou quatro semanas foram realizadas colheitas de dados que incluíram medidas de peso corporal, circunferência torácica, comprimento e largura testicular e circunferência escrotal. Foram, também, colhidos ejaculados pela eletroejaculação e avaliados os aspectos físicos e morfológicos. No momento da colheita de sêmen foram aferidas as porcentagens de desprendimento entre pênis e prepúcio e determinadas as idades médias ao desprendimento total (IDPPRE). Foram determinadas as idades médias ao aparecimento dos primeiros espermatozóides (ISEM1) e dos primeiros espermatozóides móveis (ISEM2) no ejaculado, e a puberdade seminal (IDPUB). Estabeleceram-se os índices de capacidade andrológica por pontos (ICAP). Os touros Nelore apresentaram idades médias ISEM1, ISEM2 e IDPUB de 13,1 $\pm 2,2$,

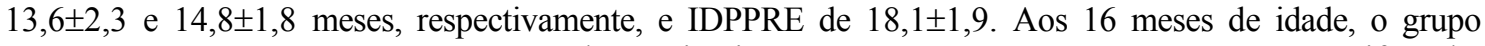
apresentou $91,4 \%, 82,6 \%$ e $73,9 \%$ dos animais com ISEM1, ISEM2 e IDPUB manifestada, respectivamente. Observou-se correlação positiva entre idade dos touros e as características de crescimento corporal, testicular, aspectos físicos dos ejaculados e correlação negativa entre a idade dos touros e os defeitos espermáticos. Observou-se correlação negativa entre a idade dos touros e a pontuação para circunferência escrotal no ICAP à puberdade $(-0,77 ; \mathrm{P}<0,001)$. Touros da raça Nelore, criados a pastos, manifestaram a puberdade precocemente antes dos 15 meses de idade. Houve um período de 51 dias desde o aparecimento dos espermatozóides no ejaculado, até a puberdade seminal. A puberdade foi mais precoce nos touros jovens com maiores testículos.
\end{abstract}

Palavras-chave: bovino, Nelore, puberdade, testículo, sêmen, circunferência escrotal

\begin{abstract}
Twenty three 10-months-old Nellore bulls, raised under pasture conditions in Brazil, were used to study puberty. Monthly measurement of scrotal circumference and testicular width and length were recorded. Semen was collected by eletroejaculation and evaluated according to physical and morphological aspects. Penile and prepuce detachment percentages were also evaluated. The ages of the presence of first spermatozoa in the ejaculate (ISEM1), first motile spermatozoa in the ejaculate (ISEM2), seminal puberty (IDPUB) and detachment between penile and prepuce (IDPPRE) were calculated. Scores of
\end{abstract}

Recebido em 2 de agosto de 2006

Aceito em 17 de novembro de 2006

E-mail: gfreneau@vet.ufg.br

Apoio: $\mathrm{CNPq} / \mathrm{FUNAPE}$ 
breeding soundness were measured. ISEM1, ISEM2, IDPUB and IDPPRE were 13.1 $2.2,13.6 \pm 2.3$, $14.8 \pm 1.8$ and $18.1 \pm 1.9$ months, respectively. At 16 months of age, the animals presented $91.4 \%, 82.6 \%$ and $73.9 \%$ with ISEM1, ISEM2 and IDPUB reached, respectively. High positive correlation were observed between age and corporal and testicular growth or seminal physical aspects. Negative correlation was verified between age and spermatic defects. High negative correlation was observed between age and score of scrotal circumference at puberty $(-0.7 ; 7 \quad P<0.001)$. Precocious puberty was observed before 15 months of age. From ISEMI to IDPUB, 51 days were elapsed. Young bulls with larger testicles reached puberty earlier.

Keywords: beef cattle, puberty, Nellore, testis, sperm, scrotal circumference

\section{INTRODUÇÃO}

A puberdade do touro é o marco inicial da fase reprodutiva e produtiva. Assim, deve ser bem conhecida, tendo-se em vista as características inerentes à raça com seu potencial genético e o ambiente onde esse potencial deverá ser expressado. A puberdade é o início da fertilidade e $\mathrm{o}$ período em que se verifica rápido desenvolvimento reprodutivo. A aplicação desses conhecimentos permite a seleção de reprodutores precoces. Existem pesquisas sobre o desenvolvimento reprodutivo puberal de bovinos de raças taurinas em ambiente de clima temperado e poucas abordaram o tema em raças zebuínas no Brasil (Godinho, 1970; Garcia et al., 1987; Dode et al., 1989; Freneau et al., 1992). Essa fase na reprodução caracteriza-se como a idade em que ocorre rápido crescimento testicular, mudanças no modelo de secreção do hormônio luteinizante, que acarreta gradual incremento da testosterona sangüínea e, como conseqüência, a iniciação da espermatogênese (Amann e Schambacher, 1983).

A puberdade é um processo lábil, que se sujeita a numerosos fatores ambientes, externos e internos, que interagem e influenciam o sistema nervoso central a modular o sistema endócrino e, por conseguinte, alterar a idade cronológica na qual o animal a manifesta (Amann e Schambacher, 1983).

As características dos ejaculados na fase puberal apresentam grande variabilidade, qualitativa e quantitativa, que foram definidas por Wolf et al. (1965). Este trabalho teve como objetivo descrever os eventos da puberdade em bovinos machos da raça Nelore, por meio dos desenvolvimentos ponderal e testicular, dos aspectos seminais e do índice de capacidade andrológica por pontos (ICAP) em touros jovens.

\section{MATERIAL E MÉTODOS}

Foram utilizados 23 tourinhos (Bos taurus indicus) da raça Nelore (sem registro) com idades entre 10 e 20 meses, em uma propriedade no município de Carmolândia, TO. Os animais foram mantidos a pasto, com várias espécies de gramíneas cultivadas, em regime extensivo, com suplementação mineral e calendário profilático de acordo com o preconizado para a região.

A cada 30 dias, os animais foram pesados individualmente e aferida a hemicircunferência torácica com auxílio de fita métrica, posteriormente multiplicada por dois, para se acompanhar o desenvolvimento corporal dos mesmos. A cada duas ou quatro semanas, foram tomadas as medidas de biometria testicular, de comprimento e largura do testículo e do perímetro escrotal, com auxílio de fita métrica e paquímetro, segundo Freneau (1991).

As colheitas de sêmen foram realizadas a cada duas ou quatro semanas, pelo método da eletroejaculação. No momento da colheita, foi avaliado o grau de desprendimento entre pênis e prepúcio. Com esses dados, foi determinada a idade média em que todos os animais apresentaram desprendimento total (IDPPRE) (Freneau et al., 1995).

Os ejaculados foram avaliados nos seus aspectos físicos (volume, motilidade e vigor espermáticos e concentração de espermatozóides por ejaculado) segundo Manual... (1998). A análise da morfologia espermática, baseada em defeitos maiores e menores, foi realizada em microscópio de contraste diferencial de interferência de fase (Lagerlof, 1934; Blom, 1973).

Foram determinadas as idades de aparecimento dos primeiros espermatozóides (ISEM1) e dos 
primeiros espermatozóides móveis (ISEM2) no ejaculado. A idade da puberdade seminal foi estabelecida no momento em que se observaram ejaculados com $10 \%$ de motilidade individual progressiva, com um mínimo de 50 × $10^{6}$ espermatozóides totais (IDPUB) (Wolf et al., 1965). Com os dados de circunferência escrotal e dos ejaculados, estabeleceram-se os índices de capacidade andrológica por pontos (ICAP) (Freneau et al., 1998).

Os dados quantitativos e qualitativos foram analisados pelo SAS (User's..., 1996) via procedimentos MEANS, FREQ, GLM. Para a comparação de médias, utilizou-se o teste Tukey. $\mathrm{Na}$ análise de freqüências, foi utilizado o método do qui-quadrado.

\section{RESULTADOS E DISCUSSÃO}

Os tourinhos apresentaram desenvolvimento corporal satisfatório, conforme demonstrado pelo peso corporal e circunferência torácica com aumentos constantes aferidos nas diferentes colheitas de dados (Tab. 1). Houve diferenças significativas dessas duas características entre as idades $(\mathrm{P}<0,001)$. Os dados indicaram que os touros, mantidos exclusivamente a pasto apresentaram ganho de peso de $0,51 \mathrm{~kg} / \mathrm{dia}$, maior que o relatado, para a mesma raça (Pinto et al., 1991). Essas observações mostraram que as condições nutricionais fornecidas aos touros foram compatíveis com o desenvolvimento reprodutivo dos mesmos.

As características de crescimento corporal e testicular e os aspectos físicos e morfológicos dos ejaculados dos touros, ao longo do período experimental, são apresentados na Tab. 1. Foram observadas diferenças entre as idades estudadas, demonstrando crescimento testicular constante, característico dessa fase em bovinos como relatado, anteriormente, em estudos com tourinhos de raças taurinas e mestiças (Freneau, 1991).

Tabela 1. Características de desenvolvimento ponderal, testicular e reprodutivo de touros Nelore criados em pasto

\begin{tabular}{|c|c|c|c|c|c|c|c|c|}
\hline & \multicolumn{8}{|c|}{ Idade (meses) } \\
\hline & 11 & 12 & 13 & 14 & 15 & 16 & 17 & 18 \\
\hline & $\overline{\mathrm{X}} \pm \mathrm{DP}$ & $\bar{X} \pm \mathrm{DP}$ & $\bar{X} \pm \mathrm{DP}$ & $\overline{\mathrm{X}} \pm \mathrm{DP}$ & $\bar{X} \pm \mathrm{DP}$ & $\bar{X} \pm D P$ & $\bar{X} \pm D P$ & $\mathrm{X} \pm \mathrm{DP}$ \\
\hline Pcorp & $178,6 \pm 15,1 \mathrm{e}$ & $191,3 \pm 17,2 \mathrm{de}$ & $204,6 \pm 22,5 \mathrm{~cd}$ & $221,2 \pm 20,9 b c$ & $238,3 \pm 20,4 b$ & $244,9 \pm 53,1 b$ & $280,7 \pm 24,1 \mathrm{a}$ & $290,7 \pm 23,9 a$ \\
\hline Ctora & $137.9 \pm 5,8 \mathrm{e}$ & $139,7 \pm 5 \mathrm{de}$ & $142,7 \pm 5,7 \mathrm{cde}$ & $144,4 \pm 6,1 \mathrm{~cd}$ & $146,9 \pm 7,3 \mathrm{bc}$ & $151,6 \pm 4,5 b$ & $156,7 \pm 4,4 a$ & $158,5 \pm 4,4 \mathrm{a}$ \\
\hline Codir & - & - & - & - & $76,6 \pm 10,2 \mathrm{c}$ & $83,0 \pm 10,2 b c$ & $90,1 \pm 9,2 \mathrm{ab}$ & $93,6 \pm 8,8 \mathrm{a}$ \\
\hline Ladir & - & - & - & - & $47,0 \pm 6,5 \mathrm{c}$ & $48,2 \pm 5,7 \mathrm{bc}$ & $52,4 \pm 5,2 \mathrm{ab}$ & $54,1 \pm 5,4 \mathrm{a}$ \\
\hline Pesc & $177,6 \pm 18 f$ & $185,7 \pm 20,4 \mathrm{f}$ & $192,6 \pm 23,4 \mathrm{fe}$ & $209,1 \pm 27,2 \mathrm{de}$ & $224,6 \pm 32,3 \mathrm{~cd}$ & $233 \pm 28,6 b c$ & $253,8 \pm 24,6 \mathrm{ab}$ & $263,5 \pm 23,2 \mathrm{a}$ \\
\hline Vol & $2,6 \pm 0,6 b$ & $2,9 \pm 0,4 \mathrm{ab}$ & $3,1 \pm 0,5 \mathrm{ab}$ & $3,1 \pm 0,4 \mathrm{ab}$ & $3,2 \pm 0,7 \mathrm{ab}$ & $3 \pm 0,7 \mathrm{ab}$ & $3,3 \pm 1,2 \mathrm{ab}$ & $3,4 \pm 0,8 \mathrm{a}$ \\
\hline Motil & $4,5 \pm 3,9 \mathrm{c}$ & $10,1 \pm 6,6 \mathrm{bc}$ & $13,1 \pm 10,4 b c$ & $21,7 \pm 15,9 b c$ & $28,2 \pm 20,6 \mathrm{ab}$ & $46,6 \pm 27,7 \mathrm{a}$ & $42,2 \pm 23,5 \mathrm{a}$ & $47,3 \pm 20,7 \mathrm{a}$ \\
\hline Vig & $1,3 \pm 0,5 \mathrm{c}$ & $0,6 \pm 1,4 \mathrm{c}$ & $1,8 \pm 0,4 \mathrm{c}$ & $1,8 \pm 0,8 \mathrm{c}$ & $2 \pm 0,8 \mathrm{bc}$ & $2,9 \pm 0,8 \mathrm{a}$ & $2,7 \pm 0,7 \mathrm{ab}$ & $2,9 \pm 0,8 \mathrm{a}$ \\
\hline $\mathrm{Sp} / \mathrm{ml}$ & $1,1 \pm 0,7 \mathrm{~b}$ & $1,9 \pm 1,4 \mathrm{~b}$ & $2,5 \pm 2,1 \mathrm{~b}$ & $4,7 \pm 4,3 b$ & $6,7 \pm 6,4 \mathrm{ab}$ & $8,5 \pm 9,7 \mathrm{ab}$ & $10,4 \pm 12,2 \mathrm{ab}$ & $15,6 \pm 15,8 \mathrm{a}$ \\
\hline $\mathrm{Sp} / \mathrm{ejac}$ & $3 \pm 2,7 b$ & $5,6 \pm 4,4 b$ & $7,4 \pm 6,4 b$ & $15,1 \pm 13,3 \mathrm{ab}$ & $21,3 \pm 19,5 \mathrm{ab}$ & $27,4 \pm 37,1 \mathrm{ab}$ & $44,4 \pm 69,4 a b$ & $61,6 \pm 74,2 \mathrm{a}$ \\
\hline Gcp & $59,5 \pm 22,8 \mathrm{a}$ & $59,9 \pm 19,4 \mathrm{a}$ & $43,0 \pm 21,7 \mathrm{abc}$ & $47,9 \pm 16,9 \mathrm{ab}$ & $37,8 \pm 18,7 b c$ & $23,7 \pm 18,3 \mathrm{c}$ & $25,7 \pm 19,1 \mathrm{c}$ & $23,7 \pm 20,5 c$ \\
\hline Dme & $12,9 \pm 2,9 \mathrm{c}$ & $16,8 \pm 2,6 \mathrm{ab}$ & $16,8 \pm 2,6 \mathrm{ab}$ & $20 \pm 3,3 \mathrm{a}$ & $13,7 \pm 3,6 b c$ & $10 \pm 3,7 d$ & $12,3 \pm 4,7 \mathrm{c}$ & $11,8 \pm 4,2 \mathrm{c}$ \\
\hline Dma & $107,9 \pm 30,3 a$ & $113 \pm 28,7 \mathrm{a}$ & $85,3 \pm 31,2 \mathrm{ab}$ & $92,8 \pm 23,9 \mathrm{ab}$ & $66,2 \pm 21,7 b c$ & $47,6 \pm 28,6 \mathrm{c}$ & $49,5 \pm 29,3 c$ & $43,3 \pm 24,6 c$ \\
\hline Dt & $120,7 \pm 31,8 \mathrm{a}$ & $129,8 \pm 28,5 \mathrm{a}$ & $102,1 \pm 32 \mathrm{ab}$ & $112,8 \pm 24 a$ & $80 \pm 22,2 b c$ & $57,6 \pm 30,1 \mathrm{c}$ & $61,9 \pm 29,9 \mathrm{c}$ & $55,1 \pm 27,4 \mathrm{c}$ \\
\hline Dpr & $33,6 \pm 15 \mathrm{~d}$ & $43,1 \pm 17,5 d$ & $52,3 \pm 23,2 \mathrm{~cd}$ & $67,8 \pm 25 b c$ & $67 \pm 25,3 \mathrm{bc}$ & $70 \pm 20 \mathrm{abc}$ & $83,9 \pm 18,1 \mathrm{ab}$ & $90,7 \pm 12,5 \mathrm{a}$ \\
\hline $\mathrm{CE}$ & $25,9 \pm 10,6 a$ & $28,7 \pm 10 \mathrm{a}$ & $27,6 \pm 12,3 \mathrm{a}$ & $25 \pm 14,5 \mathrm{a}$ & $22 \pm 13,2 \mathrm{ab}$ & $11,6 \pm 5,8 \mathrm{c}$ & $14,3 \pm 8,6 \mathrm{bc}$ & $13,2 \pm 5,7 \mathrm{bc}$ \\
\hline MOT & $1 \pm 0 \mathrm{c}$ & $1 \pm 0 \mathrm{c}$ & $1,3 \pm 0,7 \mathrm{c}$ & $2,7 \pm 3,1 \mathrm{c}$ & $4,7 \pm 5,9 b c$ & $11,1 \pm 8,3 \mathrm{a}$ & $9,3 \pm 7,5 \mathrm{ab}$ & $9,3 \pm 7,4 \mathrm{ab}$ \\
\hline MORF & $1 \pm 0 \mathrm{a}$ & $1 \pm 0 \mathrm{a}$ & $1 \pm 0 \mathrm{a}$ & $1 \pm 0 \mathrm{a}$ & $1,2 \pm 0,7 \mathrm{a}$ & $6,5 \pm 8,8 \mathrm{a}$ & $6,7 \pm 8,6 \mathrm{a}$ & $6,7 \pm 8,6 \mathrm{a}$ \\
\hline ICAP & $27,1 \pm 11 \mathrm{a}$ & $29,9 \pm 10,6 \mathrm{a}$ & $29,1 \pm 12,7 \mathrm{a}$ & $27,5 \pm 14,5 \mathrm{a}$ & $26,1 \pm 14,6 \mathrm{a}$ & $26,1 \pm 17,6 \mathrm{a}$ & $30,3 \pm 17,2 \mathrm{a}$ & $29,3 \pm 15,2 \mathrm{a}$ \\
\hline
\end{tabular}

Pcorp=peso corporal $(\mathrm{kg})$, Ctora=circunferência toráxica $(\mathrm{cm})$, Codir=comprimento do testículo direito (mm), Ladir=largura do testículo direito $(\mathrm{mm}), \mathrm{Vol}=$ volume $(\mathrm{ml})$, Pesc=circunferência escrotal $(\mathrm{mm})$, Motil=motilidade progressiva dos espermatozóides $(\%)$, Vig=vigor $(1-5), \mathrm{Sp} / \mathrm{ml}=$ espermatozóides $/ \mathrm{ml}\left(\mathrm{x} 10^{7}\right)$, ejac=Sp/ejaculado $\left(\times 10^{7}\right) \mathrm{Gcp}=$ gota citoplasmática proximal $(\%), \mathrm{Dme}, \mathrm{Dma}, \mathrm{Dt}=\mathrm{defeitos}$ espermáticos menores, maiores e totais $(\%), \mathrm{Dpr}=$ desprendimento entre pênis e prepúcio $(\%), \mathrm{CE}=$ pontuação por circunferência escrotal no ICAP, MOT=pontuação por motilidade progressiva no ICAP, MORF=pontuação por morfologia espermática no ICAP, ICAP=índice de capacidade andrológica por pontos. Valores seguidos por letras distintas na linha indicam diferenças pelo teste de Tukey $(\mathrm{P}<0,001)$

Quanto aos aspectos físicos dos ejaculados (Tab. 1), observou-se aumento progressivo na qualidade, até o final do experimento $(\mathrm{P}<0,001)$. Os aspectos da morfologia espermática (defeitos espermáticos 
maiores e totais) apresentaram queda continua ao longo das observações $(\mathrm{P}<0,001)$, porém não atingiram os patamares de qualidade de no máximo $30 \%$ fixados por Chenoweth e Spitzer (1992). Esta é uma tendência própria de touros em fase de desenvolvimento puberal, como relatado em outros estudos, tanto com raças taurinas quanto zebuínas (Wolf et al., 1965; Lunstra et al., 1978; Freneau, 1991; Brito et al., 2004). As características seminais apresentaram elevados desvios-padrão devido às diferenças na precocidade sexual entre os animais. Esse achado é característico na fase puberal de desenvolvimento reprodutivo em touros (Almquist, 1982; Amann e Schambacher, 1983). A variação no ICAP se deveu quase que exclusivamente ao crescimento testicular e não aos outros componentes do índice que aferem a qualidade seminal como a motilidade e a morfologia (Tab. 1). Essa constatação se justifica, pois o ICAP não capta as modificações seminais de touros em fase puberal e sim de touros adultos, portanto não seria ferramenta de avaliação de touros em fase puberal, sendo útil somente para identificar tourinhos mais precoces.

Observaram-se positivas e elevadas correlações entre a idade e as características de crescimento corporal e testicular e os aspectos físicos dos ejaculados, e correlação negativa com os aspectos morfológicos dos ejaculados (Tab. 2). Essa ocorrência é característica de touros na fase de desenvolvimento reprodutivo e se agrega a estudos anteriores relatados em outras raças de bovinos em climas tropical e temperado (Lusntra et al., 1978;
Freneau, 1991; Jiménez, 2002). A correlação entre circunferência escrotal e a idade, com o peso corporal e com a circunferência torácica foram similares e positivas e podem indicar a não interferência alimentar nessa fase de desenvolvimento. Condições ambientais desfavoráveis, que resultaram em atraso do desenvolvimento corporal e alteraram, também, o crescimento testicular com perda de sua relação com a idade dos animais foram relatados por Wildeus e Entwistle (1983) e Pimentel et al. (1984). O peso corporal e a circunferência torácica oferecem maiores correlações que a idade para se selecionar reprodutores com maior circunferência escrotal, independentemente dos fatores ambientais (Pimentel et al., 1984).

A circunferência escrotal foi positivamente correlacionada com os aspectos físicos dos ejaculados (motilidade progressiva, vigor $\mathrm{e}$ concentração espermática) e negativamente com os defeitos menores, maiores, totais e com a gota citoplasmática proximal $(\mathrm{P}<0,001)$. Esses fatos são característicos da fase puberal de touros das raças taurinas e zebuínas em desenvolvimento reprodutivo, tanto em ambientes tropicais quanto temperados (Lunstra et al., 1978; Rekwot et al., 1987; Madrid et al., 1988; Freneau, 1991). Essas correlações perdem valor e/ou significância, em estudos que caracterizam animais adultos, com mais de três anos de idade (Salisbury et al., 1978).

Tabela 2. Coeficientes de correlação simples entre idade e as características de crescimento corporal, testicular, aspectos físicos e morfológicos de ejaculados e índice de capacidade andrológica por pontos (ICAP) de touros Nelore em fase puberal

\begin{tabular}{|c|c|c|c|c|c|c|c|c|c|c|c|c|c|c|c|c|c|}
\hline & Idade & Pcop & Ctora & Pesc & Codir & Ladir & Vol & Turb & Motill & Vig & Conc & $\mathrm{Sp} / \mathrm{Ej}$ & Gcp & Dma & Dme & Dt & ICAP \\
\hline Idade & - & 0,91 & 0,92 & 0,86 & 0,76 & 0,77 & 0,58 & 0,16 & 0,75 & 0,70 & 0,58 & 0,56 & $-0,70$ & $-0,80$ & $-0,63$ & $-0,81$ & 0,47 \\
\hline Pcorp & 0,91 & - & 0,90 & 0,86 & 0,75 & 0,76 & 0,55 & 0,14 & 0,70 & 0,63 & 0,56 & 0,55 & $-0,62$ & $-0,72$ & $-0,57$ & $-0,73$ & 0,48 \\
\hline Ctora & 0,92 & 0,90 & - & 0,86 & 0,81 & 0,75 & 0,54 & 0,17 & 0,71 & 0,65 & 0,55 & 0,53 & $-0,63$ & $-0,72$ & $-0,59$ & $-0,74$ & 0,50 \\
\hline Pesc & 0,86 & 0,86 & 0,86 & - & 0,86 & 0,97 & 0,58 & 0,26 & 0,77 & 0,67 & 0,62 & 0,60 & $-0,70$ & $-0,78$ & $-0,60$ & $-0,79$ & 0,62 \\
\hline Codir & 0,76 & 0,75 & 0,81 & 0,86 & - & 0,88 & 39 & 0,26 & 0,66 & 64 & 54 & 0,51 & $-0,55$ & & & 67 & 0,64 \\
\hline Ladir & 0,77 & 0,76 & 0,75 & 0,97 & 0,88 & - & 0,54 & 0,35 & 0,64 & 0,50 & 0,63 & 0,63 & $-0,57$ & $-0,67$ & $-0,57$ & $-0,70$ & 0,73 \\
\hline Vol & 0,58 & 0,55 & 0,54 & 0,58 & 0,39 & 0,54 & - & 0,18 & 0,51 & 0,46 & 0,67 & 0,58 & $-0,49$ & $-0,53$ & $-0,49$ & $-0,55$ & 0,46 \\
\hline Turb & 0,16 & 0,14 & 0,17 & 0,26 & 0,26 & 0,35 & 0,18 & - & 0,26 & 0,26 & 0,79 & 0,70 & $-0,05$ & $-0,15$ & $-0,30$ & $-0,19$ & 0,71 \\
\hline Motil & 0,75 & 0,70 & 0,71 & 0,77 & 0,66 & 0,64 & 0,51 & 0,26 & - & 0,91 & 0,63 & 0,57 & $-0,76$ & $-0,83$ & $-0,62$ & $-0,84$ & 0,50 \\
\hline Vig & 0,70 & 0,63 & 0,65 & 0,67 & 0,64 & 0,50 & 0,46 & 0,26 & 0,91 & - & 0,60 & 0,56 & $-0,70$ & $-0,76$ & $-0,56$ & -0 , & 0,64 \\
\hline Conc & 0,58 & 0,56 & 0,55 & 0,62 & 0,54 & 0,63 & 0,67 & 0,79 & 0,63 & 0,60 & - & 0,96 & $-0,54$ & $-0,58$ & $-0,53$ & $-0,60$ & 0,71 \\
\hline $\mathrm{Sp} / \mathrm{Ej}$ & 0,56 & 0,55 & 0,53 & 0,60 & 0,51 & 0,63 & 0,58 & 0,70 & 0,57 & 0,56 & 0,96 & - & $-0,51$ & $-0,54$ & $-0,52$ & $-0,56$ & 0,69 \\
\hline Gcp & $-0,70$ & $-0,62$ & $-0,63$ & $-0,70$ & $-0,55$ & $-0,57$ & $-0,49$ & $-0,05$ & $-0,76$ & $-0,70$ & $-0,54$ & $-0,51$ & - & 0,95 & 0,50 & 0,93 & $-0,55$ \\
\hline Dma & $-0,80$ & $-0,72$ & $-0,72$ & $-0,78$ & $-0,65$ & $-0,67$ & $-0,53$ & $-0,15$ & $-0,83$ & $-0,76$ & $-0,58$ & $-0,54$ & 0,95 & - & 0,61 & 0,99 & $-0,55$ \\
\hline Dme & $-0,63$ & $-0,57$ & $-0,59$ & $-0,60$ & $-0,54$ & $-0,57$ & $-0,49$ & $-0,30$ & $-0,62$ & $-0,56$ & $-0,53$ & $-0,52$ & 0,50 & 0,61 & - & 0,69 & $-0,46$ \\
\hline Dt & $-0,81$ & $-0,73$ & $-0,74$ & $-0,79$ & $-0,67$ & $-0,70$ & $-0,55$ & $-0,19$ & $-0,84$ & $-0,77$ & $-0,60$ & $-0,56$ & 0,93 & 0,99 & 0,69 & - & $-0,56$ \\
\hline ICAP & 0,47 & 0,48 & 0,50 & 0,62 & 0,64 & 0,73 & 0,48 & 0,71 & 0,50 & 0,64 & 0,71 & 0,69 & $-0,55$ & $-0,55$ & $-0,46$ & $-0,56$ & - \\
\hline
\end{tabular}

Pcorp=peso corporal $(\mathrm{kg})$, Ctora=circunferência torácica $(\mathrm{cm})$, Pesc=circunferência escrotal $(\mathrm{mm})$, Codir e Ladir=comprimento e largura do testículo direito $(\mathrm{mm}), \mathrm{Vol}=$ volume ejaculado $(\mathrm{ml})$, Turb=turbilhonamento, Motil=motilidade progressiva dos espermatozóides, Vig=vigor dos espermatozóides, Conc=concentração espermática, Sp/ej=espermatozóides por ejaculado, Gcp=gota citoplasmática proximal, Dma, Dme e Dt=defeitos espermáticos maiores, menores e totais. $(\mathrm{P}<0,001)$ 
Observaram-se coeficientes de correlação elevados e negativos entre as características de aspectos físicos e morfológicos dos ejaculados. Merecem destaque as correlações negativas entre a motilidade progressiva dos espermatozóides e a gota citoplasmática proximal ou os defeitos maiores $(-0,76$ e $-0,83, \quad \mathrm{P}<0,001), \quad$ o que caracteriza a evolução dos ejaculados nessa fase de desenvolvimento, em processo de atingir a maturidade sexual. Estes dados são semelhantes aos observados em raças taurinas criadas em climas temperado e tropical (Lunstra et al., 1978; Freneau, 1991). A queda percentual da gota citoplasmática proximal pode ser usada como marcador de desenvolvimento reprodutivo de touros em crescimento.
Na Tab. 3, apresentam-se as características de peso corporal, circunferência torácica biometria testicular (comprimento e largura do testículo direito e circunferência escrotal) e de aspectos físicos e morfológicos dos ejaculados, de acordo com a idade de ocorrência dos eventos reprodutivos ISEM1, ISEM2 e IDPUB. Os espaços de tempo, transcorridos entre esses eventos, foram: 0,5 mês entre ISEM1 e ISEM2; 1,2 mês entre ISEM2 e IDPUB; 1,7 mês entre ISEM1 e IDPUB. Houve diferença $(\mathrm{P}<0,05)$ entre a IDPUB com as outras duas idades e os eventos (ISEM1 e ISEM2). Também foram observadas diferenças $(\mathrm{P}<0,05)$ entre as características de crescimento corporal, de biometria testicular e dos ejaculados. Essas diferenças caracterizariam a IDPUB e os dois outros eventos como diferentes estádios do desenvolvimento reprodutivo de touros.

Tabela 3. Características de desenvolvimento ponderal, testicular e seminal de touros Nelore a diferentes eventos reprodutivos na fase puberal

\begin{tabular}{|c|c|c|c|c|c|c|c|c|c|}
\hline \multirow[t]{2}{*}{ Evento } & \multicolumn{3}{|c|}{$\begin{array}{l}1^{\circ} \text { SPTZ no ejaculado } \\
\text { (n 23) }\end{array}$} & \multicolumn{3}{|c|}{$\begin{array}{l}1^{\circ} \text { SPTZ móvel no ejaculado } \\
\text { (n 23) }\end{array}$} & \multicolumn{3}{|c|}{$\begin{array}{l}\text { Puberdade seminal } \\
\text { (n 23) }\end{array}$} \\
\hline & $\overline{\mathrm{X}} \pm \mathrm{DP}$ & Amplitude & $\mathrm{CV}$ & $\overline{\mathrm{X}} \pm \mathrm{DP}$ & Amplitude & $\mathrm{CV}$ & $\overline{\mathrm{X}} \pm \mathrm{DP}$ & Amplitude & $\mathrm{CV}$ \\
\hline Idade & $13,1 \pm 2,2 b$ & $10-17$ & 16,5 & $13,6 \pm 2,3 b$ & $10-18$ & 16,6 & $14,8 \pm 1,8 \mathrm{a}$ & $12-18$ & 12,0 \\
\hline Pcorp & $204,6 \pm 34,7 b$ & $158-280$ & 17,0 & $211,9 \pm 37,2 b$ & $158-292$ & 17,6 & $\begin{array}{c}231,7 \pm 31,8 \\
\text { a }\end{array}$ & $193-300$ & 13,7 \\
\hline Ctora & $142,8 \pm 5,7 \mathrm{~b}$ & $134-156$ & 4,0 & $144,8 \pm 6 b$ & $134-158$ & 4,2 & $148,1 \pm 5,4 \mathrm{a}$ & $138-158$ & 3,7 \\
\hline Codir & $74,5 \pm 8,6 b$ & $57-85$ & 11,5 & $77,7 \pm 8,6 \mathrm{a}$ & $65-91$ & 11,1 & $80,5 \pm 7,9 a$ & $68-96$ & 9,8 \\
\hline Ladir & $43,7 \pm 3,9 b$ & $38-51$ & 9,0 & $45,5 \pm 3,6 \mathrm{ab}$ & $41-53$ & 7,9 & $48,1 \pm 4,1 \mathrm{a}$ & $42-55$ & 7,9 \\
\hline Pesc & $196,5 \pm 20 b$ & $165-250$ & 10,2 & $202,8 \pm 21 b$ & $165-260$ & 10,3 & $\begin{array}{c}217,4 \pm 23,1 \\
\mathrm{a}\end{array}$ & $180-270$ & 10,6 \\
\hline $\mathrm{Vol} / \mathrm{ml}$ & $2,5 \pm 0,7 \mathrm{~b}$ & $2-4$ & 25,8 & $2,8 \pm 0,9 b$ & $2-6$ & 31,4 & $3,2 \pm 0,8 \mathrm{a}$ & $2-6$ & 24,5 \\
\hline Motil & $3,3 \pm 3,5 \mathrm{c}$ & $1-10$ & 105,4 & $8,1 \pm 4,3 b$ & $2-20$ & 53,0 & $13,8 \pm 6,3 a$ & $10-35$ & 46,4 \\
\hline Vig & $1,2 \pm 0,4 \mathrm{~b}$ & $1-2$ & 36,1 & $1,3 \pm 0,6 b$ & $1-3$ & 42,5 & $1,6 \pm 0,5 \mathrm{a}$ & $1-2$ & 32,4 \\
\hline $\mathrm{Sp} / \mathrm{ml}$ & $8,5 \pm 6,6 \mathrm{~b}$ & $1-28$ & 78,1 & $14,4 \pm 7,7 \mathrm{~b}$ & $4-32$ & 53,8 & $24,6 \pm 8,8 \mathrm{a}$ & $13-49$ & 35,8 \\
\hline $\mathrm{Sp} / \mathrm{ejac}$ & $23,1 \pm 23,2 \mathrm{c}$ & $2-112$ & 100,6 & $42,3 \pm 29,3 b$ & $8-114$ & 69,4 & $76,5 \pm 27,7 a$ & $50-147$ & 36,3 \\
\hline Gcp & $60,3 \pm 20,2 a$ & $19,5-89$ & 33,4 & $49,1 \pm 21,7 b$ & $19,5-85,5$ & 44,3 & $37,0 \pm 14,9 \mathrm{c}$ & $19,5-65,5$ & 40,3 \\
\hline Dme & $13,4 \pm 3,2 \mathrm{a}$ & 8-19 & 23,6 & $14,4 \pm 3,7 \mathrm{a}$ & $8-24$ & 26,1 & $16,5 \pm 4,1 \mathrm{a}$ & $8-22,5$ & 24,9 \\
\hline Dma & $108,4 \pm 26,9 \mathrm{a}$ & $55-140,5$ & 24,9 & $93,6 \pm 30,5 a$ & $48-142$ & 32,5 & $74,3 \pm 22,7 b$ & $40-123$ & 30,6 \\
\hline Dt & $121,8 \pm 28,7 \mathrm{a}$ & $64-159$ & 23,5 & $108 \pm 31 \mathrm{a}$ & $56-155,5$ & 28,7 & $90,8 \pm 24,7 b$ & $56-144,5$ & 27,1 \\
\hline Dpr & $39,56 \pm 14,9 \mathrm{c}$ & $10-60$ & 37,7 & $47,5 \pm 19,5 b$ & $10-80$ & 41,0 & $60,4 \pm 20 \mathrm{a}$ & $20-100$ & 33,2 \\
\hline $\mathrm{CE}$ & $25 \pm 13,8 \mathrm{a}$ & $1-40$ & 55,2 & $24,8 \pm 13,1 \mathrm{a}$ & $5-40$ & 53,2 & $25,2 \pm 14,2 \mathrm{a}$ & $5-40$ & 56,2 \\
\hline MOT & $1 \pm 0$ & 1 & 0 & $1 \pm 0$ & 1 & 0 & $1,1 \pm 0,4$ & $1-3$ & 38,4 \\
\hline MORF & $1 \pm 0$ & 1 & 0 & $1 \pm 0$ & 1 & 0 & $1,1 \pm 0,4$ & $1-3$ & 38,3 \\
\hline ICAP & $27,0 \pm 13,8 \mathrm{a}$ & $3,0-42,0$ & 51,1 & $26,8 \pm 13,2 \mathrm{a}$ & $7,0-42,0$ & 49,2 & $27,4 \pm 14 a$ & $7-42$ & 51,2 \\
\hline
\end{tabular}

Pcorp=peso corporal $(\mathrm{kg})$, Ctora=circunferência toráxica $(\mathrm{cm})$, Codir=comprimento do testículo direito (mm), Ladir=largura do testículo direito $(\mathrm{mm})$, Pesc $=$ circunferência escrotal $(\mathrm{mm}), \mathrm{Vol}=$ volume ejaculado $(\mathrm{ml})$, Motil=motilidade progressiva dos espermatozóides $(\%)$, Vig=vigor (1-5) Sp/ml=espermatozóides $/ \mathrm{ml}\left(\times 10^{6}\right), \mathrm{Sp} / \mathrm{ejac}=\mathrm{Sp} /$ ejaculado $\left(\times 10^{6}\right), \mathrm{Gcp}=$ gota citoplasmática proximal $(\%)$, Dme, Dma e $\mathrm{Dt}=$ defeitos espermáticos menores, maiores e totais $(\%)$, Dpr=desprendimento entre pênis e prepúcio (\%), CE=pontuação por circunferência escrotal no ICAP, MOT=pontuação por motilidade progressiva no ICAP, MORF=pontuação por morfologia espermática no ICAP, ICAP=índice de capacidade andrológica por pontos. Valores seguidos por letras distintas na linha indicam diferenças pelo teste Tukey $(\mathrm{P}<0,05)$. 
Os intervalos entre idades a eventos reprodutivos da fase puberal foram constantes e comparáveis aos relatados em estudos de animais taurinos e mestiços (Wolf et al., 1965; Oyedipe et al., 1981; Rekwot et al., 1987; Freneau, 1991). A puberdade, em geral, começa com o aparecimento da fertilidade, marcada por diferentes eventos (Amann e Schambacher, 1983). Esses eventos não ocorrem simultaneamente e, devido a isso, diferentes autores utilizaram combinação de diversos parâmetros para caracterizar o início da puberdade. Assim, a IDPUB foi definida por Wolf et al. (1965), como a idade do primeiro ejaculado com $10 \%$ de motilidade progressiva e 50 milhões de espermatozóides totais, tendo encontrado correlações de 0,55 e 0,75 entre IDPUB e as ISEM1 e ISEM2.

Neste estudo, os espaços de tempo transcorrido entre as ISEM1, ISEM2 e IDPUB foram mais prolongados que os relatados nas raças taurinas em clima temperado (Wolf et al., 1965; Lunstra et al., 1978; Freneau, 1991), revelando possível diferença genética e/ou ambiental entre as subespécies taurina e zebuína. Entretanto, quando esses tempos são comparados aos registrados anteriormente em animais zebus, as diferenças em favor dos animais deste estudo foram grandes, o que indica melhor adaptação às condições edafo-climáticas da região, aliada, provavelmente, à maior precocidade da raça Nelore (Godinho, 1970; Oyedipe et al., 1981; Rekwot et al., 1987; Vieira et al., 1988; Dode et al., 1989). Freneau et al. (1992) observaram em animais da raça Gir, criados sob condições semiextensivas, com suplementação energética durante sua criação, espaços de tempo comparáveis aos deste estudo.

As idades médias ISEM1, ISEM2 e IDPUB foram $13,1 \pm 2,2, \quad 13,6 \pm 2,3$ e $14,8 \pm 1,8$ meses, respectivamente. Essas idades foram maiores que as relatadas em raças taurinas (Wolf et al 1965; Lunstra et al 1978, Almquist, 1982; Pruitt et al., 1986). Estes resultados quando comparados com aqueles de touros Holandeses e seus mestiços $F_{1}$, em condições tropicais, essas diferenças se estreitam para 75 dias (Freneau, 1991; Jiménez, 2002).

Foram observadas elevadas amplitudes fenotípicas em todas as características estudadas neste trabalho. As médias registradas foram marcadamente menores que as verificadas em animais de raças zebuínas e de mestiças com taurinas (Oyedipe et al., 1981; Rekwot et al 1987; Garcia et al., 1987; Vieira et al., 1988; Dode et al., 1989; Freneau et al 1992; Silva-Mena, 1997; Brito et al., 2004). Em razão da grande amplitude nas idades em que houve manifestação da puberdade, existe espaço para realizar-se pressão de seleção sobre a precocidade.

Os touros Nelore, deste estudo, se encontram como os mais precoces criados em regime extensivo. Especificamente dentro da raça Nelore, esses animais foram mais precoces, em mais de cinco meses, em relação aos estudados por outros autores, também em regime de pastagem (Dode et al., 1989; Silva et al., 1991). Em estudo realizado por Brito et al., (2004), os animais foram divididos em precoces e tardios, a média de idade à puberdade, observada por esses autores, para animais precoces e tardios diferiu em 2,7 e em 7,6 meses respectivamente, em relação à média encontrada neste experimento e em 5,2 meses quando se compararam a média geral de ambos os estudos. A circunferência escrotal à puberdade (Tab. 3) foi $21,7 \mathrm{~cm}$ e próxima à relatada para animais precoces $(22,5 \mathrm{~cm})$ por Brito et al. (2004). Essas diferenças observadas podem ser atribuídas à qualidade das pastagens ou, simplesmente, à maior precocidade pela seleção de touros doadores do sêmen utilizado na produção dos animais deste experimento. Unanian e Silva (1996) relataram, em touros Nelore, a puberdade seminal aos 12 meses de idade em alguns animais, o que também foi constatado neste estudo, entretanto, esse resultado poderia ser justificado pelo fato de se tratar de animais de elite (PO), além de não constar, no trabalho dos referidos autores, o regime de criação adotado.

Os resultados deste trabalho assemelham-se aos publicados em tourinhos de raças taurinas $\mathrm{e}$ mestiças $F 1$, no que se refere à baixa qualidade dos ejaculados, próprios da fase puberal na espécie bovina. Esse fato deveria ser considerado na avaliação de touros jovens, nessa fase, para a identificação de indivíduos precoces e não como critério de descarte.

As características de crescimento corporal, biometria testicular e dos ejaculados apresentaram diferenças $(\mathrm{P}<0,05)$ entre a IDPUB e as ISEM1 e ISEM2 (Tab. 3).

Na Fig. 1, são apresentadas as freqüências dos eventos puberais de acordo com a idade dos 
animais. Observou-se que aos 16 meses de idade, $91,4 \%, 82,6 \%$ e $73,9 \%$ dos touros manifestaram ISEM1, ISEM2 e IDPUB, respectivamente. Como observado na comparação das médias (Tab. 3), a IDPUB apresentou-se como evento distanciado das ISEM1 e ISEM2 $(\mathrm{P}<0,05)$, apesar de manterem evolução similar entre os três. A idade ao desprendimento entre pênis e prepúcio ocorreu mais tardiamente. Nas ISEM1, ISEM2 e IDPUB, os touros apresentaram desprendimento de $39,6 \pm 14,9, \quad 47,5 \pm 19,5$ e $60,4 \pm 20,0$ com diferenças significativas entre eles $(\mathrm{P}<0,05)$.
A idade de desprendimento entre o pênis e o

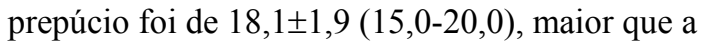
observada em touros holandeses e mestiços F1 (Freneau et al., 1995). Apesar de o desprendimento total entre as mucosas ter sido observado em todos os animais é possível que essa característica seja determinada em menor idade, pois nas colheitas de ejaculado, a exposição do pênis não ocorreu em todos os animais.

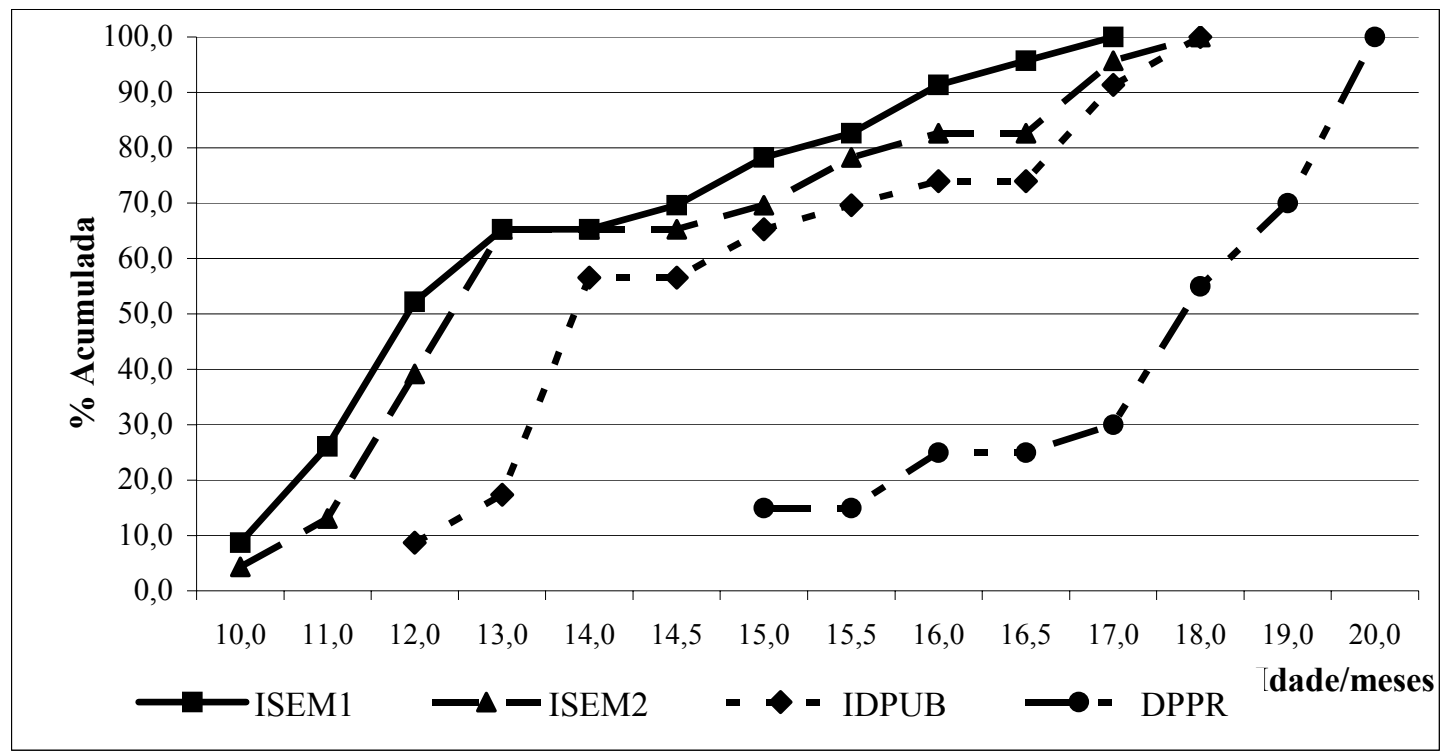

Figura 1. Freqüências dos eventos reprodutivos da puberdade, de acordo com a idade, em touros Nelore criados em pasto.

$\mathrm{Na}$ análise do ICAP, verificou-se que, pelas características seminais puberais terem sido de baixa qualidade, o maior aporte de pontos foi dado pela circunferência escrotal sendo quase insignificante em relação à morfologia e à motilidade espermática progressiva. Assim, para circunferência escrotal à puberdade, houve elevado coeficiente de correlação negativo entre a idade à puberdade e os pontos dentro do ICAP $(-0,77 ; \quad \mathrm{P}<0,0001)$, o que se assemelha aos resultados de estudos anteriores em raças taurinas nos quais animais de maior tamanho testicular foram mais precoces (Lunstra et al., 1978).

Na Tab. 4, observa-se que o percentual de touros, nas diferentes idades, aumentou entre as faixas do ICAP, apresentando-se o primeiro touro na faixa $\mathrm{A}$, aos 20 meses de idade $(4,4 \%)$. Na faixa $\mathrm{D}$, o percentual de animais variou de $91,4 \%$ a $21,8 \%$ entre 15 e 20 meses de idade. Como os touros não alcançaram índices de qualidade de sêmen que indicassem terem atingido a maturidade sexual, conforme relatado anteriormente por Freneau et al. (1998), o ICAP seria uma ferramenta muito mais importante para identificar touros pela sua precocidade, do que para descarte de animais nessa fase de desenvolvimento.

Nas condições em que este estudo foi conduzido, pode-se concluir que touros da raça Nelore, criados a pasto, manifestaram a puberdade antes dos 15 meses de idade. Entre a ISEME1 e a IDPUB transcorreramse, em média, 51 dias. Touros jovens com maior circunferência escrotal apresentaram menor idade à puberdade. Devido à amplitude de variação das idades na manifestação da puberdade entre os animais estudados, verificou-se condição para se fazer uma pressão de seleção quanto à precocidade sexual. 
Tabela 4. Faixas do índice de capacidade andrológica por pontos (ICAP) na fase puberal de touros Nelore criados em pasto

\begin{tabular}{ccccccccc}
\hline Faixa do ICAP & \multicolumn{2}{c}{$\mathrm{A}$} & \multicolumn{3}{c}{$\mathrm{B}$} & \multicolumn{2}{c}{$\mathrm{C}$} & \multicolumn{2}{c}{$\mathrm{D}$} \\
\hline Idade em meses & $\mathrm{n}$ & $\%$ & $\mathrm{n}$ & $\%$ & $\mathrm{n}$ & $\%$ & $\mathrm{n}$ & $\%$ \\
\hline 15 & - & - & - & - & 2 & 8,70 & 21 & 91,35 \\
16 & - & - & 1 & 4,35 & 2 & 8,70 & 20 & 87,00 \\
17 & - & - & 1 & 4,35 & 6 & 26,4 & 16 & 69,60 \\
18 & - & - & 1 & 4,35 & 7 & 30,45 & 15 & 65,25 \\
19 & - & - & 2 & 8,70 & 11 & 47,85 & 10 & 43,50 \\
20 & 1 & 4,35 & 8 & 34,80 & 9 & 39,15 & 5 & 21,75 \\
\hline
\end{tabular}

Diferença entre colunas $\chi^{2}(\mathrm{P}<0,05)$

\section{AGRADECIMENTOS}

À Fazenda Vale do Boi que cedeu os animais e as instalações para este experimento. A Breno Nahuel pela digitação do trabalho.

\section{REFERÊNCIAS BIBLIOGRÁFICAS}

ALMQUIST, J.O. Effect of long term ejaculation at high frequency on output of sperm, sexual behavior, and fertility of Holstein bulls; relation of reproductive capacity to high nutrient allowance. J. Dairy Sci., v.65, p.814-823, 1982.

AMANN, R.P.; SCHAMBACHER, B.D. Physiology of male reproduction. J. Anim. Sci., v.57, suppl., p.380-403, 1983.

BLOM, E. The ultrastructure of some characteristics sperm defects and a proposal for a new classification on the bull spermiogram. Nord. Vet. Med., v.25, p.383-391, 1973.

BRITO, L.F.; SILVA, A.E.; UNANIAN, M.M.; et al. Sexual development in early and latematuring Bos indicus and Bos indicus x Bos taurus crossbred bulls in Brazil. Theriogenology,. v.62, p.1198-1217, 2004.

CHENOWETH, P.J.; SPITZER, J.C. A new Bull Breeding Soundness Evaluation form. Proc. Society of Theriogenology, AGM, p. 63-70, 1992.

DODE, M.A.N.; SCHENK, J.A.P.; SILVA, A.E.D.F. Determinação da puberdade em machos Nelore e Mestiços. Rev. Bras. Reprod. Anim., v. 1, supl., p.185, 1989.

FRENEAU, G.E.; GUIMARÃES，J.D.; VALE FILHO, V.R. Despreendimento puberal entre pênis e prepúcio em touros europeus e mestiços
F1. Rev. Bras. Reprod. Anim, v.19, p.153-160, 1995.

FRENEAU, G.E.; GUIMARÃES, J.D.; VALE FILHO, V.R. et al. Pubertal and post-pubertal development in Gyr zebu bulls in Brazil. In: INTERNATIONAL CONGRESS OF ANIMAL REPRODUCTION, 12., 1992. The Hague. Proccedings... The Hague, 1992. p.1981-1984.

FRENEAU, G.E. Desenvolvimento reprodutivo em tourinhos holandeses e mestiços holandêsgir, desde os seis até os 21 meses de idade (Puberdade e pós-puberdade). 1991. 254f. Dissertação (Mestrado em Medicina Veterinária) - Escola de Veterinária, Universidade Federal de Minas Gerais, Belo Horizonte.

FRENEAU, G.E; PUOLI, J.R.; DIAS, F.M.G.N. Capacidade andrológica por pontos em touros Nelore: resultados após quatro de anos de aplicação em MS. In: REUNIÃO ANUAL DA SOCIEDADE. BRASILEIRA DE ZOOTECNIA, 35., 1998, Botucatu. Anais... Botucatu: SBZ, 1998. p.149-151.

GODINHO, H.P. Puberdade em bovinos Gir estimada pela análise de sêmen. Arq. Esc. Vet. $U F M G$, v.22, p.165-169, 1970.

GARCIA, J.M.; PINHEIRO, L.E.L.; OKUDA, H.T. Body development and semen physical and morphological caracteristics of young Guzera bulls. Ars. Vet., v.3, p.47-53, 1987.

JIMÉNEZ, S. H. Sexual development in dairy bulls in Mexican tropics. Theriogenology, v.58, p.921-932, 2002.

LAGERLOF, N. Morphologische Untersuchungen uber. Veranderim spermabild und in den Hoden bei Bulle mit Verminderter oder auf gehobe ver Fertilitat. Acta Pathol. Microbiol. Scand., v.1, suppl. XIX, 1934. $254 \mathrm{f}$. 
LUNSTRA, D.D.; FORD, J.J.; ECHTERKAMP, S.E. Puberty in beef bulls: Hormone cocentration, growth, testicular development, sperm production and sexual aggressiveness in bulls of different breeds. J. Anim. Sci., v.46, p.1054-1062, 1978.

MADRID, N.; OTT, R.S.; VEERAMACHANEMI RAO, D.N. et al. Scrotal circumference, seminal characteristics, and testicular lesions of yearling Angus bulls. Am. J. Vet. Res., v.49, p.579-585, 1988.

MANUAL para exame andrológico e avaliação de semen animal. Belo Horizonte: CBRA, 1998. 49p.

OYEDIPE, E.O.; KUMI DIAKA, J.; OSORI, D.I.K. Determination of onset of puberty in zebu bulls under tropical conditions of Northern Nigeria. Theriogenology, v.16, p.419-430, 1981.

PIMENTEL, C.A.; FERREIRA, U.M.M.; MORAES, J.C.F. et al. Desenvolvimento testicular e corporal em touros de corte. Rev. Bras. Reprod. Anim., v.8, p.27-33, 1984.

PINTO, P.A.; LÔBO, R.B.; MADUREIRA, E.H. Seleção de tourinhos zebus, da raça Nelore, para reprodução, às idades de 12, 18 e 24 meses. Correlações da circunferência escrotal (CE) com peso dos animais. In: REUNIÃO ANUAL DA SOCIEDADE BRASILEIRA DE ZOOTECNIA, 28., 1991, João Pessoa. Anais....João Pessoa: SBZ, 1991. p.447.

PRUITT, R.J.; CORAH, L.R.; STEVENSON, J.S. et al. Effect of energy intake after weaning on the sexual development off beef bulls. II. Age at first mating, age at puberty, testosterone and scrotal circumference. J. Anim. Sci., v.63, p.579585,1986

REKWOT, P.I.; OYEDIPE, E.O.; AKEREJOLA, O.O. et al. The effect of protein intake on the onset of puberty in of Bunaji and Frisian $x$ Bunaji crossbred bulls in Nigeria. Theriogenology, v.28, p.427-33, 1987.

SALISBURY, G.W.; VANDEMARK, N.L.; LODGE,J.R. Fisiologia de la reproducción e inseminación artificial de los bovidos. 2.ed. Zaragoza: Acribia, 1978. 831p.

SILVA, F.A.E.D.; DODE, M.A.N.; ROSA, A.A.M. et al. Estabelecimento da atividade sexual em machos da raça Nelore no Brasil Central. In: CONGRESSO BRASILEIRO DE REPRODUÇÃO ANIMAL, 9., 1991, Belo Horizonte. Anais... Belo Horizonte: CBRA, 1991. p. 425.

SILVA-MENA, C. Peripubertal traits of Brahman bulls in Yucatan. Theriogenology, v.48, p.675-685, 1997.

UNANIAN, M.M.; SILVA, A.E.D.F. Estudo da precocidade reprodutiva em machos da raça Nelore. In: CONGRESSO BRASILEIRO DE MEDICINA VETERINÁRIA, 24., Goiânia. Anais... Goiânia, 1996. p.111.

USER'S guide: statistics. Version 6.03. Cary, NC: SAS Institute, 1996. 530f.

VIEIRA, R.C.; ALENCAR, M.M.; ESTEVES, S.N. Efeito da suplementação alimentar sobre o comportamento reprodutivo de tourinhos Canchin. Pesq. Agropec. Bras., v.23, p.97-102, 1988.

WILDEUS, S.; ENTWISTLE, K.W. A quantitative histological study of testicular and epedidymal development in Bos indicus cross bulls. Anim. Reprod. Sci., v.6, p.1-10, 1983.

WOLF, F.R.; ALMQUIST, J.O.; HALE, E.B. Prepuberal behavior and pubertal characteristics of beef bulls on high nutrient allowance. J. Anim. Sci., v.24, p.761-764, 1965. 